\title{
Studio comparativo delle aeque potabili del Cantone Ticino, dal punto di vista ehimico e dal punto di vista microbiologico
}

di
A. Verda.
(9. X, 19.)

Il Cantone Ticino può essere diviso, in riguardo alla geologia dei bacini imbriferi delle sue acque in alcune zone più o meno ben delimitate.

Se noi apriamo una carta geologica non troppo dettagliata, per es. quella di Schmidt (Geolog. Kartenskizze der Alpen zwischen St. Gotthardt und Mont Blanc) o quella di Taramelli (Carta geologica della regione dei tre laghi), noi vediamo come il Cantone Ticino sia diviso in due zone principali, la cui delimitazione corrisponde press' a poco al confine naturale formato dallo spartiacque delle montagne della linea del Ceneri, che divide il cantone in due regioni tra loro relativamente dissimili, il Sopra- ed il Sottoceneri. Nella zona superiore noi troviamo in forte prevalenza l'ortogneiss, che forma uno strato relativamente compatto ed uniforme di tutti i massicci delle regioni locarnesi, della Valle Maggia, delle parti inferiori delle valli di Blenio e Leventina, mentre le parti superiori di queste. due valli e specialmente il massiccio del St. Gottardo e quello dell' Adula sono costituiti da gruppi di roccie diverse in cui i micaschisti si alternano con roccie calcaree del Trias sparse tra $\mathrm{i}$ paragneiss diversi et le filliti ed amfiboliti, senza dimenticare le zone gessose di Val Bedretto e dei dintorni di Airolo. Nella zona inferiore noi troviamo prevalenza dei micaschisti cristallini, eccezione fatta solo per gli estremi lembi del cantone, al sud di Lugano, dove i terreni sono formati da zone diverse alternantesi di rocee cristalline con rocee calcaree, seguite poi da zone alluvionali.

Nel Sopraceneri inferiore solo la riva sinistra del Lago Maggiore col massiccio del Tamaro è formata da mieaschisti cristallini, per cui il confine reale geologico delle due zone principali 
su esposte oltrechè dalle montagne del Ceneri sarebbe formato dal Lago Maggiore. La divisione reale fra queste due zone è data in ultima analisi dalla zona alluvionale del piano di Magadino. E pure importante di notare come a nord del piano di Magadino nelle zone che si estendono dal Camoghè parallelamente alla linea formata dalla catena dei monti della riva sinistra del Ticino, sulla riva destra del fiume stesso, abbia principio una varietà speciale di gneiss analoga alla diorite ed al gabbro, che sviluppandosi sulla riva destra del Lago Maggiore arriva fino ad Ivrea nel Piemonte, formando la cosidetta "Zona di Ivrea".

Se si toglie questa particolarità del gneiss del Bellinzonese e del Locarnese, i massicci del Sopraceneri sono di una uniformità quasi assoluta, rotta solo in piccoli punti da alcuni minimi banchi calcarei e divisa dalle zone alluvionali dei fiumi, del Ticino, del Brenno, della Moesa e della Morobbia. Una uniformità analoga si constata poi nella regione del Ticino meridionale dal Ceneri fino al Lago di Lugano, dove dominano gli schisti cristallini, a dire vero, in un certo numero di varietà, di colore e di consistenza e divisi dai giacimenti alluvionali principali del Vedeggio e del Cassarate, oltrechè dai sedimenti meno importanti di torrenti. Si constatano solo alcune particolarità a Manno, dove si riscontrano piccoli giacimenti di carbone e di aragonite. Poco a nord-est di Lugano, cominciano le zone calcaree formate dalla linea dei Denti della Vecchia, del Boglia, del Monte Bré, che poi si sviluppano a sud del lago nel massiccio giurassico del Generoso. A sud di Lugano le rocce calcaree si alternano con le masse porfiriche, per terminare nelle zone alluvionali del Mendrisiotto e dopo una breve zona di conglomerati subalpini, formati dalle colline di Pedrinate e di Monte Olimpino, nonchè daì colli a sud di Como, si estendono poi le immense distese alluvionali della Lombardia.

Oltre alle varie zone suddescritte che possono influenzare la natura specialmente chimica delle acque potabili ticinesi, si dovrà tener conto nello studio della natura dei terreni dei bacini inbriferi, di alcune accidentalità locali del terreno, come depositi morenici più o meno vasti, depositi alluvionali localizzati, frane antiche o recenti.

Volendo fare una classificazione delle acque potabili del Cantone Ticino, non sara possibile (volendo tener conto di tutti i 
fattori che possono influenzare la composizione chimica ed un poco anche la composizione batteriologica delle acque) di seguire le divisioni geografiche, che corrispondono alle varie zone geologiche suddescritte. Però basta il semplice esame delle circostanze suesposte per dimostrare già la possibilità di dividere le acque del cantone Ticino in quattro grandi gruppi principali, che sarebbero i seguenti :

I. Acque della zona degli ortogneiss (Valli sopracenerine).

II. Acque della zona dei micaschisti (Valli del Luganese).

III. Acque dêlle zone calcaree (Parte meridionale del distretto di Lugano, parte montuosa del distretto di Mendrisio, alcune acque della regione del Gottardo).

IV. Acque dei terreni di alluvione (Acque del sottosuolo delle basse valli, piani di Magadino e del Vedeggio, basso Mendrisiotto, acque dei terreni morenici).

In base al tenore delle acque in sostanze minerali ed in sostanze alcaline, le acque del cantone Ticino possono pure essere divise in quattro grandi gruppi approssimativi, che corrispondono nella maggior parte dei casi ai quattro grandi gruppi geologici succitati :

I. Acque aventi un tenore di materie minerali inferiore o di poco superiore a $50 \mathrm{mg}$. per litro ed un' alcalinità inferiore a $1 \mathrm{~cm}^{3} \mathrm{n}$. HCl per litro (Acque sgorganti da rocce di ortogneiss);

II. Acque aventi un tenore di materie minerali tra 50 e $100 \mathrm{mg}$. per litro ed un' alcalinità superiore ad 1 ed inferiore a $3 \mathrm{~cm}^{3}$ n. HCl per litro (Acque da rocce di micaschisti);

III. Acque calcaree aventi un tenore di materie minerali superiore a $200 \mathrm{mg}$. ed un' alcalinità superiore a $5 \mathrm{~cm}^{3} \mathrm{n} . \mathrm{HCl}$ per litro (Acque provenienti da zone dolomitiche, da Lias e Trias calcarei);

IV. Acque di composizione chimica intermedia fra quelle suesposte con tenore di alcalinità variabili (Acque provenienti da detriti morenici e da terreni alluvionali).

Si capisce come in questi quattro gruppi troveranno posto la maggior parte delle acque del nostro Cantone, quantunque alcune eccezioni non mancheranno ed in conseguenza di accidentalità geologiche dei terreni, alcune acque non potranno essere classificate con sufficiente precisione. Cosi sono specialmente da 
notare le acque sulfuree alcaline del Ritom, quelle di Acquarossa, alcune acque minerali di Val Bedretto ecc.

Nei gruppi suddescritti si osservano però alcune anomalie dipendenti da accidentalità del terreno o da infiltrazioni da zone diverse, infiltrazioni dall' humus, dai detriti superficiali ecc. Si nota pure sempre una certa differenza anche nelle stesse zone tra acque profonde ed acque superficiali. Queste ultime si avvicinano naturalmente nella loro composizione chimica alle acque del quarto gruppo, pur contenendo un tenore di sostanze organiche generalmente più elevato. Mentre le acque provenienti direttamente dalla roccia sono da considerare come acque piovane accumulate in serbatoi naturali, le acque di regioni moreniche o provenienti da detriti superficiali o dall' humus hanno subito una macerazione di queste sostanze e l'influenza della filtrazione è buona o cattiva a seconda che la porosità del terreno è tale da ritenere o da cedere sostanze organiche all' acqua.

Composizione chimica delle acque.

Sulla composizione chimica delle acque ticinesi si può osservare quanto segue:

In generale la maggior parte delle acque ticinesi sono caratterizzate da deboli coefficienti di mineralizzazione, ciò che rappresenta indubbiamente un vantaggio per la loro potabilità. Anche le acque sgorganti da zone calcaree sono relativamente povere in residui calcinati. Questi residui raggiungono assai raramente i $300 \mathrm{mg}$. per litro. Si tratta in generale di rocee assai compatte e di acque non ricche di acido carbonico libero, ciò che impedisce la formazione di forti dosi di bicarbonati alcalini. Solo le acque solfuree-alcaline della regione del Ritom e quelle di Ossasco in Val Bedretto presentano dei residui minerali elevatissimi con dosi forti di solfati.

Un altra caratteristica comune all' immensa maggioranza delle acque ticinesi è l' assenza di nitrati anche nelle acque relativamente impure. Le materie organiche presenti in queste acque danno certamente luogo a formazione di ammoniaca, ma in assenza di sali minerali agenti come catalizzatori o per la mancanza di basi saline e di ioni elettro-positivi l' ammoniaca non si ossida neppure parzialmente. E pure raro di trovare dei nitriti anche in acque notoriamente assai impure. 
L'ammoniaca per contro si riscontra assai frequentemente nelle nostre acque ed anche nelle acque normali i dosaggi dell' ammoniaca sono soggetti ad una certa irregolarità, tantochè noi abbiamo dovuto rinunciare al dosaggio quantitativo dell' ammoniaca libera come base di apprezzazione delle acque. Cosi mentre acque impure erano relativamente povere in ammoniaca, acque batteriologicamente pure ne avevano delle dosi elevate.

Tale anomalia deve, secondo noi, essere attribuita alla relativa impermeabilità dei terreni, oltrechè alla deficienza di materie organiche sia nel terreno che nelle acque.

Anche il tenore delle materie organiche delle nostre acque e un criterio molto malsicuro nel giudizio della potabilità delle acque del Cantone Ticino, perchè numerose sono le acque riconosciute inquinate che diedero dei tenori bassi di materie organiche. E tale constatazione fu da noi fatta sia col metodo dell'ossidazione col permanganato di potassio, sia col metodo del calcolo del materia organica dalla differenza tra residui secchi e calcinati (Glühverlust). Il terreno poco permeabile e povero di materie organiche, impedisce la solubilizzazione delle materie organiche contenute già in dose relativamente piccola nei nostri terreni silicei. L' acqua di pioggia scorre fra le roccie e si sprofonda fra le anfrattuosità delle medesime, per uscire poi in altri punti sottostanti, senza aver assorbito materie organiche in quantità rilevante. D'altra parte una purificazione autogena dell' acqua stessa qualora essa sia stata per caso inquinata è poco probabile. Nel terreno poco poroso, manca l' ossigeno e forse anche mancano i catálizzatori necessari all' ossidazione della materia organica.

Questa ha per contro tendenza, anche se si riscontra in dose non elevata di dar luogo a formazione di ammoniaca, che non può ossidarsi che assai difficilmente. Ciò spiega in pari tempo anche l' assenza quasi generale di nitrati et di nitriti nelle nostre acque.

Un caso tipico al riguardo di tali fenomeni è dato dalle acque del lago di Muzzano, che sebbene ricchissime di detriti vegetali in decomposizione sono state da noi riconosciute ancora recentemente come prive di nitrati e di nitriti e con un tenore di materie organiche in soluzione (dosate nell' acqua filtrata) relativamente basso. Non sarà senza interesse a più riguardi di conoscere 
la composizione chimica di quest' acqua interessantissima, che anche nei periodi atmosferici più tranquilli è normalmente torbida per la presenza di un quantitativo ingente di detriti organici in sospensione.

Acqua del laghetto di Muzzano.

Alcalinità ín $\mathrm{cm}^{3} \mathrm{n}$. IICl $. \quad 1,4$ per litro

\begin{tabular}{|c|c|c|c|c|c|c|c|c|c|}
\hline $\mathrm{CaCO}_{3}$ & .. & & . & .7 & & ng. & per litro & & \\
\hline Residuo & secco & . & . & .9 & & $n$ & $"$ & (Aequa & filtrata \\
\hline id. & calcinato & - & & .8 & & $"$ & $n$ & $n$ & ” \\
\hline Materie & organiche (G & lüh & verlus & ast) 1 & & " & n & $n$ & $n$ \\
\hline Ossidabi & ilità . . & & & & & $n$ & $”$ & $"$ & $n$ \\
\hline Materie & org. calcol. & . & . & .3 & & $n$ & $\eta$ & $n$ & " \\
\hline Durezza & totale & • & . & 8 & & & & & \\
\hline " & temporanea & & & & & & & & \\
\hline y & permanente & & . . & . . .1 & & & & & \\
\hline
\end{tabular}

Ammoniaca libera

$\mathrm{Cl}$

$\mathrm{SO}_{4}$

$\mathrm{NO}_{2}$

$\mathrm{NO}_{3}$

$\mathrm{Fe}$
Presente

0 (tracce nel residuo organico)

0

0

0 (o traccie infinitesimali)

traccie

Esame microscopico del sedimento = Presenza di detriti vegetali, plancton, alghe, infusori (llagellati e cigliati), Beggiatoa alba.

Le constazioni suesposte concernenti l'ammoniaca, le sos-r tanze organiche, i nitrati ed i nitriti delle nostre acque si riferiscono all' immensa maggioranza delle acque stesse sgorganti da terreni silicei ed anche in gran parte a quelle provenienti da roccie dolomitiche. Per quanto concerne le acque calcaree di queste roccie notiamo che esse hanno un tenore di materie minerali relativamente più debole di quelle provenienti da banchi calcarei meno compatti.

Il comportamento delle acque pluviali nei terreni alluvionali è un pò diverso da quello suddescritto. Nei terreni alluvionali del basso Ticino (Mendrisiotto), delle vallate del Ticino e del Vedeggio le acque di sottosuolo sono certamente per un tempo più lungo a contatto col terreno ed il terreno più poroso permette da una parte una maggior solubilizzazione delle materie organiche $\mathrm{e}$ dall'altra una migliore filtrazione ed una più completa ossidazione 
delle sostanze organiche. Però siamo sempre anche qui in presenza di sabbie silicee, aventi un potere di filtrazione certamente ottimo ma per contro poco addatte alla solubilizzazione delle materie organiche, per mancanza di sali minerali. Tali sabbie sono certamente le più addatte a funzionare come filtri naturali delle acque.

Cosi le acque del sottosuolo del Vedeggio usate dalla città di Lugano, pur avendo un tenore di materie organiche leggermente superiore a quello delle acque di sorgente sono purissime e quasi totalmente libere da microorganismi e quelle del sottosuolo del fiume Ticino, usate dalla città di Bellinzona, sono pure sufficientemente potabili, quantunque captate in condizioni non del tutto superiori ad ogni critica, e tale da dare alle acque stesse delle differenze di composizione notevolissime.

In vicinanza di Giubiasco, per contro, in vicinanza di un pozzo nero, un acqua captata ad una profondità di circa $20 \mathrm{~m}$ conteneva un tenore di materie organiche assai elevato. E probabile che la mancanza di catalizzatori ritardi l'ossidazione delle materie organiche che pure sono dai terreni sabbiosi ben filtrate.

Un tenore più elevato di materie organiche si riscontra nelle acque sotterranee del terreno argilloso del Mendrisiotto ed in quelle della zona del Monte Generoso. Queste ultime sono specialmente degne di nota. Abbiamo qui una stratificazione dei banchi calcarei in modo tale che l'acqua scorre tra $i$ vari strati rocciosi in cui si trova uno straterello di limo ed argilla. Acque di sorgenti anche profondissime si trovano qui ricche di materia organica e presentano frequenti fenomeni d'intorbidamento.

Nelle roccie di micaschisti di zone poco elevate e molto umide, per es. nelle colline dei dintorni di Lugano, incontriamo delle roccie friabili, lamellari (sapel), nelle quali aumenta il tenore delle materie organiche ed in certa proporzione anche quello delle materie minerali delle acque sotteranee.

Sono pure ricche in materia organica le acque sgorganti da regioni speciali con torbiere (cosi a Coldrerio, a Genestrerio), e quelle provenienti da una regione speciale, quale la zona dei conglomerati di Pedrinate. 
Neppure in queste acque aventi dosi un po elevate di materie organiche, si trovano però delle dosi notevoli e spesso neppure dosi apprezzabili di nitrati. Per la formazione dei nitrati come si verifica nelle acque dell' altipiano svizzero o delle regioni del Giura, occorre una energica ossidazione dell' azoto delle sostanze organiche, che per le speciali condizioni dei nostri terreni non si verifica quasi mai nel Cantone Ticino. Le acque dei nostri laghi che pur sono alimentati da torrenti e ruscelli e fiumi provenienti da zone geologiche svariate sono assolutamente prive di nitrati, anche nei punti dove esse non sono direttamente inquinate. La presenza di nitrati nelle acque del nostro paese è quindi segno quasi certo di inquinamento.

Interessante è pure l' esame comparativo delle acque ticinesi in rapporto al loro tenore in cloruri. Solo le acque della terza zona (zona calcarea) contengono piccole quantità di cloruri. Tracce minime sono pure contenute neile acque della quarta zona (zona di alluvione o morene). In generale anche nelle acque calcaree il tenore dei cloruri è inferiore a $10 \mathrm{mg}$ per litro e possono essere considerate come eccezioni quelle che contengano $20 \mathrm{mg}$. Questa cifra limite stabilita dal Manuale svizzero delle derrate alimentari non ha alcuna importanza per il giudizio delle nostre acque. La ricerca ed eventualmente il dosaggio dei cloruri hanno per contro un gran valore per noi nel giudizio delle acque, perchè la presenza di traccie di cloruri nelle acque delle prime due zone, sia in quelle della zona cristallina (ortogneiss), sia in quelle sgorganti dal micaschisti è per lo più indizio di sospetto.

Per le altre acque è difficile fissare delle cifre limiti, ma non si è lungi dalla verità stabilendo un tenore massimo di $5 \mathrm{mg}$. per le acque della zona alluvionale e di $10 \mathrm{mg}$. per quelle della zona calcarea.

Per terminare I' esame della composizione chimica aggiungeremo alcune tabelle concernenti la composizione chimica delle acque dei vari tipi summenzionati, una circa la composizione dell'acqua del sottosuolo del fiume Ticino (a Bellinzona), una• del sottosuolo del Vedeggio, una del lago di Lugano, l' acqua della Breggia e di sorgenti sgorganti dalla regione adiacente (acque Chiasso), inoltre quantunque non attinenti in modo diretto al nostro argomento, ma a titolo di curiosità, l' analisi dell' acqua del lago Ritom e dei suoi affluenti. 
Analisi batteriologica dell'acqua del Lago di Lugano (Lab.chimico Zurigo)

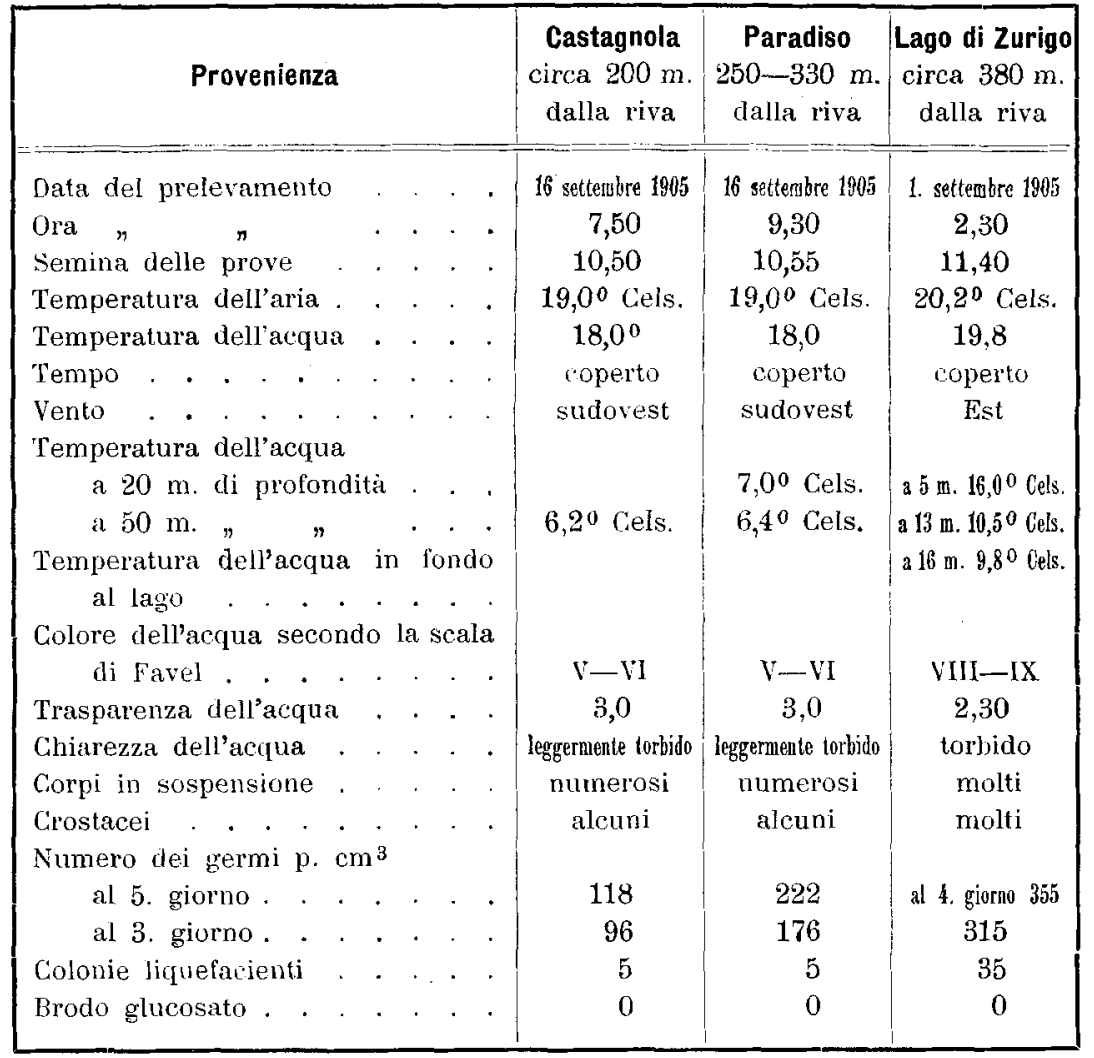

Analisi chimica dell'acqua del Lago di Lugano.

\begin{tabular}{|c|c|c|c|c|c|}
\hline \multicolumn{3}{|l|}{ Provenienza } & Castagnola & Paradiso & Lago di Zurigo \\
\hline Residuo secco e calcinato & mg. & litro & 140,0 & 138,0 & \\
\hline Alcalinità & $"$ & 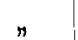 & 110,0 & 109,5 & 121,5 \\
\hline Ossidabilità & $"$ & $n$ & 9,08 & 2,15 & 3,98 \\
\hline Materie organiche & $"$ & $"$ & 40,0 & 10,7 & 19,9 \\
\hline Ammoniaca & $n$ & $"$ & 0 & 0 & 0,004 \\
\hline Ammoniaca albuminoide & $"$ & $"$ & 0,018 & 0,026 & 0,060 \\
\hline Nitriti & $n$ & $r$ & 0 & 0 & 0 \\
\hline Nitrati & $"$ & $n$ & 0 & 0 & 0 \\
\hline Cloruri & $"$ & $"$ & 4,2 & 4,2 & 5,09 \\
\hline Solfati & $"$ & 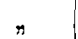 & tracce & tracce & tracee \\
\hline Durezza temporanea & & & $11,0^{0}$ & $10,95^{0}$ & $12,15^{\circ}$ \\
\hline
\end{tabular}


Acqua del Lago Maggiore, prelevata a $100 \mathrm{~m}$. dalla riva di piazza Giardino in Locarno ad una profonditc் $d i \quad 8 \mathrm{~m}$.

(12 agosto 1919)

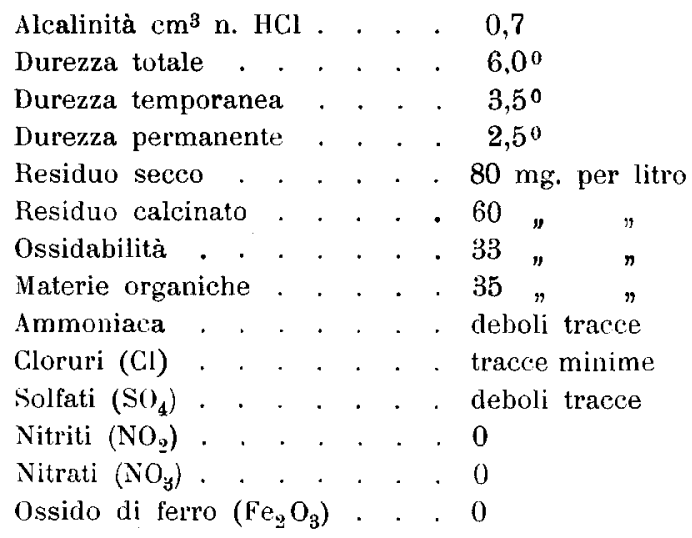

Acqua del sottosuolo Bellinzona, terreno allwionale del Ticino.

(15 luglio 1918)

Temperature alla sorgente: Aria $20^{\circ}$, Acqua $12^{\circ}$, suolo $15^{\circ}$.

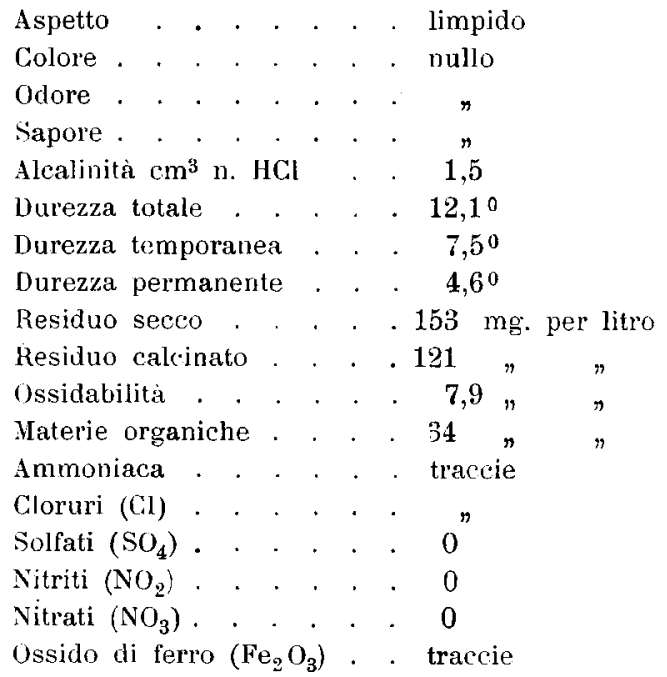

Acqua del soltosuolo del Vedeggio, terreno alluvionale.

Temperature alla sorgente: Aria $5^{0}$, Acqua $10^{\circ}$, Suolo $5^{0}$.

Aspetto . . . . . . limpido

Colore . . . . . . . nullo

Odore . . . . . . . "

Sapore . . . . . . . . "

Alealinita $\mathrm{em}^{3}$ n. HCl . . . 0,8

Durezza totale . . . . 5,30 
Durezza temporanea . . . $4,0^{\circ}$

Durezza permanente . . . 1,30

Residuo secco . . . . . $80 \mathrm{mg}$. per litro

Residuo calcinato . . . 53 n "

Ossidabilitâ . . . . 2,1 n $n$

Materie organiche. . . 10,5 "

Ammoniaca . . . . . 0

Cloruri (Cl) . . . . . . tracce minime

Solfati $\left(\mathrm{SO}_{4}\right), \ldots . . . \quad 0$

Nitriti $\left(\mathrm{NO}_{2}\right)$. . . . . . 0

Nitrati $\left(\mathrm{NO}_{3}\right)$. . . . . . 0

Ossido di ferro $\left(\mathrm{Fe}_{2} \mathrm{O}_{3}\right) \cdot$. 0

Acqua Chiasso N. 1 alla camera di sedimentazione.

(22 agosto 1919)

\begin{tabular}{|c|c|c|c|c|c|}
\hline Aspetto & & . & . & limpido & \\
\hline Colore . . & . & . & . & nullo & \\
\hline Odore & . & . & . & normale & \\
\hline Sapore . . & . . . & . & . & $\eta$ & \\
\hline Alcalinità $\mathrm{cm}^{3}$ & 3 n. $\mathrm{HC}$ & & . & 2,8 & \\
\hline Durezza totale & e. . & . & . & $15,5^{0}$ & \\
\hline Durezza temp & oranea & . & . & $14,0^{\circ}$ & \\
\hline Durezza perm & lanente & . & . & . $\quad 1,5^{0}$ & \\
\hline Residuo seceo & & . & & . $180 \mathrm{mg}$. & per litro \\
\hline Residuo calcir & nato. & . & . & $.155 \quad$ n & $\pi$ \\
\hline Ossidabilità . & & & . & . 4,36 & $n$ \\
\hline Materie organ & iche & . & . & . 21,84 & $n$ \\
\hline A mmoniaca. & $\cdot$ & . & . & tracce & \\
\hline Cloruri (Cl) . & . & . & . & presenti & \\
\hline Solfati $\left(\mathrm{SO}_{4}\right)$. & . & • & & 0 & \\
\hline Nitriti $\left(\mathrm{NO}_{3}\right)$ & . & . & $\cdot$ & 0 & \\
\hline
\end{tabular}

Acqua del fiume Breggia

(22 agosto 1919)

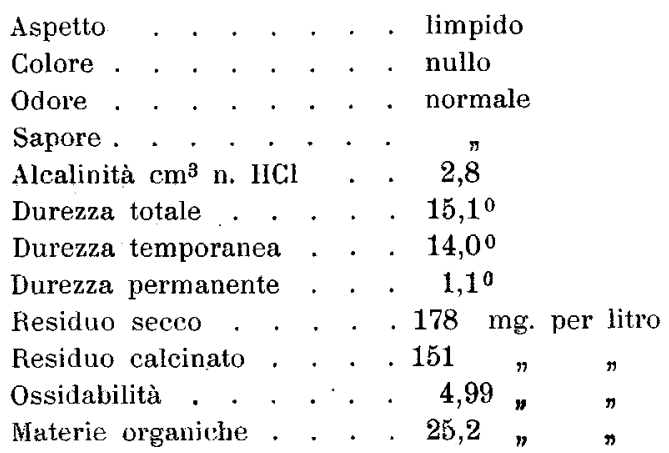


$\begin{array}{llllllll}\text { Ammoniaca } & . & \cdot & . & . & . & . & \text { traccie } \\ \text { Cloruri }(\mathrm{Cl}) & . & . & . & . & . & . & \text { pressenti } \\ \text { Solfati }\left(\mathrm{SO}_{4}\right) & . & . & . & . & . & . & 0 \\ \text { Nitriti }\left(\mathrm{NO}_{2}\right) & \cdot & . & . & . & . & . & 0\end{array}$

\section{Acqua del Ritom}

(7 gennaio 1917)

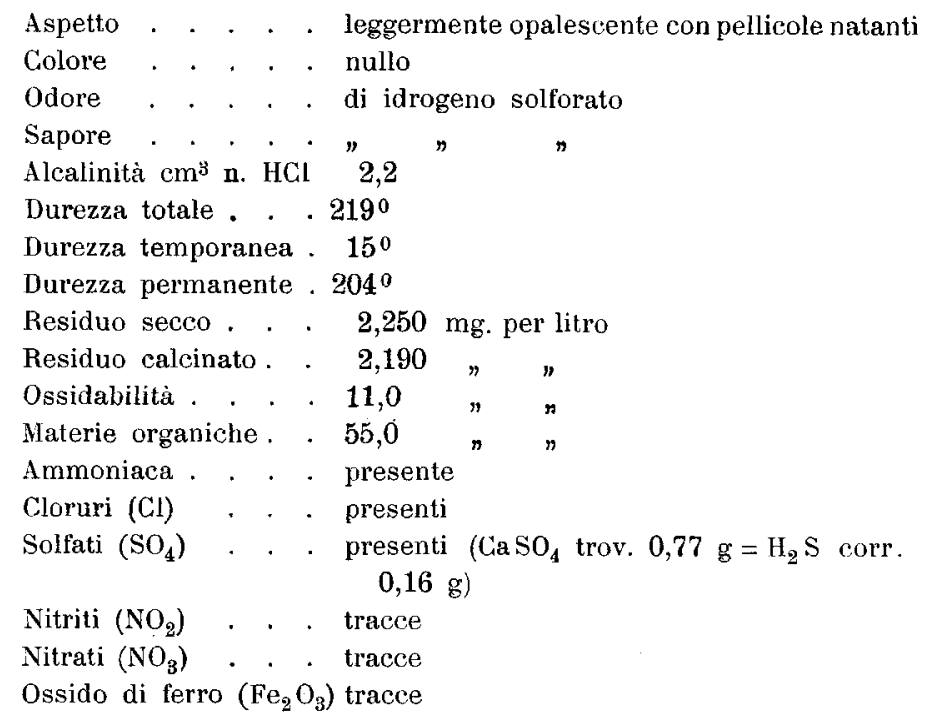

Analisi dell'acqua degli affluenti del Ritom $I$.

\begin{tabular}{|c|c|c|c|c|c|}
\hline & $\begin{array}{c}\text { Sorgente } \\
\text { presso } \\
\text { Ritom } \\
\text { mg. }\end{array}$ & $\begin{array}{l}\text { Scolo } \\
\text { lago } \\
\text { Tom }\end{array}$ & $\begin{array}{c}\text { Sorgente } \\
\text { tra Ritom } \\
\text { e Campo } \\
\text { mg. }\end{array}$ & $\begin{array}{l}\text { Sorgente } \\
\text { S. Cario } \\
\text { mg. }\end{array}$ & $\begin{array}{c}\text { Sorgente } \\
\text { Campo-Riva } \\
\text { destra } \\
\text { mg. }\end{array}$ \\
\hline Residuo secco & 410 & - & 757 & 1149 & 1179 \\
\hline Residuo calcinato & 395 & 一 & 747 & 1104 & 1052 \\
\hline Alcalinità $\mathrm{cm}^{3} \mathrm{n} . \mathrm{HCl}$ & 1,9 & - & 1,7 & 1,7 & 1,7 \\
\hline Durezza totale . & $39,5^{\circ}$ & 一 & 74,70 & $110,4^{0}$ & $105,2^{0}$ \\
\hline Durezza temporanea & $9,5^{0}$ & - & $8,5^{0}$ & $8,5^{0}$ & $8,5^{\circ}$ \\
\hline Durezza permanente & $30,0^{0}$ & - & $66,2^{\circ}$ & $101,9^{\circ}$ & $96,7^{\circ}$ \\
\hline Ammoniaca . . & tracce & tracce & tracce & tracce & tracce \\
\hline Nitrati . . . & 0 & 0 & 0 & 0 & 0 \\
\hline Cloruri. . . . & 21,0 & 0 & tracce & 0 & tracee \\
\hline Solfato di calcio. & 261,0 & 0 & 598 & 828 & 880 \\
\hline $\mathrm{SO}_{4} \cdot \cdot \cdot \cdot$. & 184,0 & 0 & 422 & 585 & 622 \\
\hline $\mathrm{CaCO}_{3} \cdot$. . & 95,0 & 0 & 85 & 85 & 85 \\
\hline
\end{tabular}


Analisi dell'acqua degli affuenti del Ritom II.

\begin{tabular}{|c|c|c|c|c|c|}
\hline & $\begin{array}{c}\text { Scolo } \\
\text { lago } \\
\text { Cadagno } \\
\mathrm{mg} .\end{array}$ & $\begin{array}{c}\text { Mulinascia } \\
\mathrm{mg} .\end{array}$ & $\begin{array}{c}\text { Affluente } \\
\text { Malinaseiz } \\
\text { in Campo } \\
\text { mg. }\end{array}$ & $\begin{array}{c}\text { Canariscic } \\
\text { di } \\
\text { Campo } \\
\text { mg. }\end{array}$ & $\begin{array}{c}\text { Canariscio } \\
\text { di } \\
\text { Ritom } \\
\text { mg. }\end{array}$ \\
\hline Residuo secco . . & 270 & 100 & 85 & 23 & 37 \\
\hline Residuo calcinato & 250 & 87 & 80 & 20 & 32 \\
\hline Alcalinità $\mathrm{cm}^{3} \mathrm{n} . \mathrm{HCl}$ & 1,0 & 1,2 & 1,5 & 0,3 & 0,3 \\
\hline Durezza totale . . & 25 & 8,7 & 8,0 & 2,0 & 3,2 \\
\hline Durezzo temporanea & 5 & 6,0 & 7,5 & 1,5 & 1,5 \\
\hline Durezza permamente & 20 & 2,7 & 0,5 & 0,5 & 1,7 \\
\hline Ammoniaca : : & tracce & tracce & tracee & tracce & tracce \\
\hline Nitrati . & 0 & 0 & 0 & 0 & 0 \\
\hline Cloruri . . . & 0 & 0 & 5 & 0 & 0 \\
\hline Solfato di calcio. & 19,5 & tracce & 0 & 0 & 0 \\
\hline $\mathrm{SO}_{4} . . . .$. & 14,0 & tracce & 0 & 0 & 0 \\
\hline $\mathrm{CaCO}_{3}$ & 50,0 & 60,0 & 75 & 15 & 15 \\
\hline
\end{tabular}

Flora batteriologica delle acque ticinesi.

Dal punto di vista microbiologico, noi possiamo osservare nella massima parte delle acque potabili del Cantone Ticino, il fenomeno corrispondente al debole tenore in materia organica delle acque medesime. Il numero dei germi delle acque da noi esaminate anche in regioni imbrifere certamente mquinate, eccezione fatta per i pozzi fortemente inquinati od in relazione diretta con fonti di inquinamento, è sempre relativamente basso ed il numero delle colonie che si sviluppano su gelatina al terzo giorno è raramente superiore ai 200-300 per centimetro cubo, e per lo più anche in acque superficiali non arriva alla cifra di 100, che è indicata nel Manuale svizzero delle Derrate alimentari, come base di giudizio delle acque potabili. Per il giudizio delle acque potabili del nostro cantone noi dobbiamo aver ricorso ad altri criteri un pò più complessi.

Certamente le nostre acque sono, per la loro deficienza di certi sali minerali, per la loro mancanza di fosfati, la debole alcalinità predominante, la tenue dose di sali calcarei e specialmente per la scarsità delle materie organiche, dovuta per lo più allo strato di humus assai ridotto che ricopre il nostro suolo nella più gran parte delle nostre regioni, un cattivo terreno di coltura per i microorganismi, che quivi si sviluppano assai difficilmente. 
Noi dobbiamo dare una maggiore importanza alle variazioni del numero dei germi che si constatano in vari periodi dell' anno, dopo le pioggie, dopo i temporali. In alcune località noi abbiamo potuto seguire le curve delle precipitazioni atmosferiche e vedere la corrispondenza quasi perfetta coll' aumento del numero dei germi delle acque potabili. In un' acqua di buona qualità la costanza del numero dei germi è data dalla circostanza della costante filtrazione delle acque di pioggia nei terreni, prima di arrivare ai depositi imbriferi. L' aumento delle precipitazioni atmosferiche può bensi accelerare il fenomeno di filtrazione naturale delle acque, ma la diluzione dovuta all' aumento del volume dell' acqua compensa largamente qualche deficienza di filtrazione.

Un esempio caratteristico di questa costanza da noi osservata ora da oltre cinque anni è dato dalle acque del sottosuolo del Vedeggio usate dalla città di Lugano. Queste acque vengono da noi regolarmente sottoposte ad analisi batteriologica ogni trimestre ed il numero dei germi in esse trovato non ebbe mai a superare i 20. Queste acque sono captate con pozzi artesiani, che vanno fino ad una profondità di $45 \mathrm{~m}$ nella sabbia silicea da cui è costituito il piano alluvionale del Vedeggio. Un migliore strato filtrante potrebbe difficilmente riscontrarsi in natura.

Assai diverso si presenta per contro il pozzo scavato a circa 20-25 metri nel sottosuolo del Ticino dalla città di Bellinzona. Qui noi abbiamo delle variazioni nel numero dei germi che vanno da 10 fino ad oltre 100 per $\mathrm{cm}^{3}$. Oltre alla minor profondità dei pozzi, la diversa natura della sabbia del piano alluvionale del Ticino ha certo un' influenza su questo fenomeno. Qui abbiamo pure delle sabbie silicee, ma più ricche in elementi calcarei provenienti dalle zone calcaree del massiccio del Gottardo. Inoltre si deve notare come il livello dell' acqua del sottosuolo bellinzonese si alzi assai più di quello del sottosuolo del Vedeggio, specialmente per il maggior numero di affluenti che si versano nel Ticino a Nord di Bellinzona. Le condizioni locali del pozzo di Bellinzona sono pure non troppo felici, data la vicinanza della caserma. L' assenza del Colibacillo anche nelle acque del sottosuolo di Bellinzona, permette però di ritenere anche quest' acqua se non proprio batteriologicamente purissima, almeno sufficientemente pura perchè possa ancora essere utilizzata come acqua potabile, pure con un'accurata sorveglianza del suo funziona- 
mento. L' insufficienza quantitativa delle acque del sottosuolo bellinzonese producendo forti divergenze nel livello del pozzo nei periodi di magra è però un fatto che può esercitare una certa influenza anche sul tenore quantitativo dell' acqua in microorganismi.

Il numero dei germi è pure soggetto a variazioni frequenti nelle acque calcaree della zona del Generoso. Rilievi fatti all' aćquedotto comunale di Chiasso hanno dimostrato come in poco più di un mese nel 1913 il numero dei germi ha potuto variare da 280 a 560 per discendere a 310 poco più di una settimana dopo, mentre appunto la portata dell' acquedotto variava da 100 litri circa al minuto secondo fino a 350 per ricadere bruscamente fino agli 80 litri al minuto. Il Dr. Mollet, Geologo militare, ha constatato, che tale periodo di variazioni parallele nel numero dei germi e nello spaccio delle sorgenti corrispondeva appunto ad un aumento rapido delle precipitazioni osservate all'osservatorio del Monte Generoso preceduto da un periodo asciutto e seguito da una settimana di mancanza di pioggie.

Fenomeni analoghi potrebbero essere constatati in molte acquedotti della regione del Lias calcareo. Noi potremmo citare Mendrisio, Muggio, Castel S. Pietro ecc.

Una particolarità della roccia sfaldata in lunghi crepacci del Monte Generoso è anche di contenere tra le fenditure della rocca un leggero strato argilloso quasi sempre umido. Quando le precipitazioni aumentano fortemente, queste acque trascinando seco dell' argilla s'intorbidano e per le materie organiche racchiuse nell' argilla, il numero dei germi aumenta considerevolmente. Non per questo tutte queste acque sono senz' altro da condannare. Il giudizio definitivo dovrà basarsi sulla possibilità o meno che le sfaldature della roccia siano inquinate da stalle od abitati.

Per dare un' idea generale del tenore delle acque del nostro Cantone, pur ammettendo che tali dati statistici abbiano un valore assai relativo, indicato specialmente dalla casualità dei prelevamente che le circostanze ci impongono di eseguire, non sarà senza interesse di osservare come nel 1918 sopra circa 300 analisi batteriologiche di acque esaminate, il numero dei germi sviluppatisi al terzo giorno era inferiore à 50 per $\mathrm{cm}^{3}$ in oltre $50 \%$ dei casi, nel 10 per cento dei casi era inferiore a 100, nel 20 per cento dei casi era inferiore a 500 e nel 20 per cento dei casi 
superiore a 500. Le acque furono ciò nondimeno ritenute non potabili nel $42 \%$ dei casi.

Dal punto di vista del numero dei germi e facendo astrazione dalla potabilità e dal punto di vista strettamente igienico sottoposto frequentemente a piccole contingenze locali, si può dire che le acque più pure si hanno nelle regioni a zone alluvionali, ed in quelle a roccie cristalline. Un tenore di germi intermedio si trova nella regioni del micaschisto. Più elevato "è il numero dei germi nei terreni formati dal lias et trias calcareo e nei terreni argillosi.

Dal punto di vista qualitativo noi possiamo osservare come una importanza non indifferente spetti nel giudizio delle acque potabili alla presenza di colonie liquefacienti la gelatina, mentre la presenza di colonie fluorescenti ed anche la presenza di muffe sono dovute ad accidentalità senza importanza per il giudizio complessivo delle sorgenti. Per quanto la distinzione trà colonie liquefacienti e colonie non liquefacienti la gelatina, alla quale è stato riconosciuto un certo valore da Robert Koch nell' esame delle acque di Berlino fin dal 1882, sia ora stata contradetta da molti autori, noi abbiamo l'impressione che una tale distinzione non sia senza valore per le acque del nostre Cantone. L'importanza di tale distinzione dipende certamente dalla flora qualitativa regionale ed è possibile che laddove i bacilli liquefacienti banali ed innocui non sono troppo diffusi nell' aria, la presenza di tali bacilli nell' acqua dipenda probabilmente da infiltrazioni mentre cosi non è dove si riscontrano varietà numerose di microbi. Diciamo però che una ricerca sistematica ed intenzionale al riguardo non è stata da noi fatta ed il nostro giudizio si basa su impressioni riportate è vero da un gran numero di analisi, ma che potrebbero anche essere accidentali o particolari a certe acque e non applicabili alle acque dell' intero nostro cantone.

Lo stesso può dirsi sulla circostanza del numero delle varietà delle specie microbiche da noi trovate nelle acque del nostro Cantone. Il numero delle varietà ci sembra un concetto non indifferente per il giudizio delle acque del nostro Cantone e la teoria di Migula ci sembra avere da noi un certo valore.

La ricerca del colibacillo nelle acque del Cantone Ticino. ci ha dimostrato che questo bacillo è presente con una certa 
frequenza in piccole dosi nelle acque del nostro Cantone, anche in casi in cui non vi è motivo di contestazione dell' acqua. Per dimostrare tale presenza noi abbiamo in un grandissimo numero di acque esaminate prelevati campioni di oltre $100 \mathrm{~cm}^{3}$ e seminate sul brodo lattosato concentrato $A b b a$ tutto il residuo delle colture su gelatina, dunque circa $100 \mathrm{~cm}^{3}$ di acqua. Cosi abbiamo potuto con una certa frequenza dimostrare l' esistenza nell' acqua di bacilli del gruppo del Coli, che davano se non tutte, almeno la massima parte delle reazioni del Colibacillo tipico Escherich quindi produzione di acido lattičo, coagulamento del latte, spesso formazione di indolo, sviluppo di gaz ecc., oltre ad avere $\mathbf{i}$ caratteri microscopici del Colibacillo. Certamento si devone condannare tutte le acque nelle quali il Colibacillo o dei bacilli coliformi si possano rivelare in piccole dosi per esempie con colture di $1 \mathrm{~cm}^{3}$ di acqua. E noi possiamo in base all' esperienza nostra confermare che la prova all' agar rosso neutro, con $1 \mathrm{~cm}^{3}$ sia essa atta a dimostrare il colibacillo, sia che essa indichi la presenza di un certo gruppo di microorganismi di origine animale o più specialmente di origine intestinale, quando essa sia nettamente positiva con sviluppo di gaz e produzione della fluorescenza verde caratteristica, ha un reale valore nel giudizio delle acque potabili. Per l' esperienza nostra, essa concorda, più ancora che il numero dei germi con le constatazioni fatte da noi alle sorgenti od alle prese ed ora possiamo aggiungere anche con una serie di oltre 200 sopraluoghi eseguiti durante il periodo della mobilitazione di guerra da una schiera di geologi militari nel nostro Cantone con l'esame di alcune migliaja di sorgenti. Essa rimane la prova pratica la più semplice della purezza di un' acqua, pur non avendo essa naturalmente un valore assoluto e specialmente quando essa sia negativa, non essendo esclusa la possibilità che l'acqua momentaneamente pura dal punto di vista batteriologico possa poi inquinarsi successivamente.

L' aggiunta di agar-agar al rosso neutro, è certamente praticamente di impiego assai più facile e vantaggioso che non le soluzioni di rosso neutro in brodo glucosato o lattosato, con l'uso di speciali apparecchi e tubi per poter meglio constatare lo sviluppo di gaz. Noi riteniamo questo mezzo solido di coltura come quello che risponde attualmente il meglio ai bisogni della pratica. 
Da un punto di vista assai più generale dobbiamo ancora osservare come, sia pure in via eccezionale, siano possibili inquinamenti delle acque oltrechè per infiltrazioni dirette alle sorgenti nel terreno, anche a traverso l' aria infetta da polviscolo proveniente da latrine, letamaj, scoli di acque luride non canalizzate ecc. $\mathrm{E}$ un punto che viene assai spesso dimenticato quando si passi all' esame di acquedotti, e sul quale non è inutile fermarci qualche istante. Noi abbiamo potuto constatare in comuni dotati di acque di sorgenti purissime, come acque di robinetti o di serbatoj, fossero dimostrate inquinate ed assai dissimili nelle loro reazioni specialmente all' analisi batteriologica dall' acqua che arrivava ad essi, pur essendo esclusa qualsiasi soluzione di continuità delle condotte. La vicinanza di letamaj, latrine a secco, anche se poco utilizzate, scoli aperti di lavatoj ecc. a serbatoj o fontane, non dovrebbe essere tollerata, anzi tale circostanza dovrebbe essere specialmente notata nei casi in cui si constatino delle malattie infettive in qualche comune.

E certamente verità elementare nota a tutti, anche a profani in materia che un giudizio sicuro di un' acqua potabile non è possibile che un seguito ad accurato esame di tutto l'impianto che adduce l'acqua, della natura del terreno delle sorgenti o delle prese, delle relazioni di queste località con locálità abitate od esistenza di stalle ecc. Ma un fatto che non abbiamo trovato citato nei testi da noi consultati, che si occupano di esame e perizia di acque potabili, è la dimostrazione diretta delle infiltrazioni di acque superficiali nelle prese o serbatoj a mezzo della constatazione di depositi calcarei sulle pareti, con formazione di striscie o macchie bianche, e specialmente col formarsi di vere e proprie stalattiti di carbonato di calcio. I profani e persino degli ingegneri ritengono che tali formazioni siano dovute alla formazione di salnitro, quale si constata nei luoghi umidi, ma pur lasciando da parte il fatto che qualora vi fosse in un serbatojo una tale produzione di vapori ammoniacali od azotati da produrre formazione di nitrati, già saremmo in presenza di un ambiente inquinato, noi abbiamo sempre constatato che tali striscie, macchie o formazioni di stalattiti sono dovute a carbonato di calcio, ciò che prova che siamo in presenza di acque non provenienti dall' evaporazione od umidità interna, ma da acque provenienti dall' esterno della presa o serbatojo attraverso al cemento delle 
prese o serbatoj, quindi a vere e proprie infiltrazioni di acque superficiali.

Una questione importante che è spesso dibattuta fra gli igienisti è quella di sapere se si debba attribuire maggiore importanza all' analisi chimica od all' analisi batteriologica delle acque e quale concordanza si riscontri fra i risultati della prima e quelli della seconda. Già numerosi autori si occuparono di tale questione. Ancora recentemente un chimico francese che ebbe a specializzarsi nell'analisi delle acque potabili delle zone di guerra pose nuovamente tale questione allo studio. Il Dr. L.C. Maillard basandosi sopra numerose esperienze, mise in evidenza la superiorità del metodo chimico, che da risultati in tempo assai più breve del metodo batteriologico, è meno soggetto all' aleatorio del caso dei prelevamenti, alle variazioni accidentali e darebbe, quando si conoscano bene $\mathrm{i}$ caratteri delle acque potabili insospettate di una regione, risultati più attendibili. Questo autore ${ }^{1}$ ) conchiude:

I. Quando il metodo chimico dichiara un' acqua sospetta l' analisi batteriologica diviene inutile,

II. Se un' acqua è risultata comparativamente buona all' analisi chimica, l' analisi batteriologica non potrà dichiararla cattiva,

III. Raramente si constata una divergenza fra le conclusioni delle due analisi nel senso di una severità maggiore dell' analisi batteriologica.

Noi non possiamo affatto sottoscrivere alle due ultime conclusioni, perchè risulta dalle numerose analisi da noi eseguite sia dal punto di vista chimico che batteriologico (alcune migliaja), che nel nostro Cantone la quasi totalità delle acque rispondono ai requisiti generalmente ammessi per l'analisi chimica delle acque, pur essendovene una buona percentuale che vennero da noi all' analisi batteriologica ed all' esame dei luoghi dichiarate sospette o pericolose. Inoltre le variazioni dei componenti chimici, anche nei limiti ammessi per le buone acque di una data regione e di un dato terreno imbrifero, rivelano solo assai tardivamente gli inquinamenti che invece risultano dalla presenza di un numero esagerato di germi o dalla presenza del colibacillo in proporzioni tali da far condannare un' acqua. Specialmente il rapporto tra le

1) Bull. Acadćm. Médecine t. 79, ํo 9, séance du 5 mars 1918. 
materie organiche e la composizione batteriologica di un' acqua potabile deve essere ritenuto inattendibile, poichè noi abbiamo dimostrato la possibilità che acque anche fortemente inquinate mostrino un tenore di materie organiche assai basso.

E specialmente all' esame dei luoghi che tale circostanza ha potuto essere messa in luce, in modo da togliere ogni dubbio al riguardo.

Lugano, Laboratorio cantonale d' Igiene.

\section{Die elektrochemische Oxydation des Benzaldehyds und der Benzoësäure voll}

Fr. Fiehter und Eldor Uhl.

(18. X. 19.)

\section{Das Problem.}

Beim Vergleich der elektrolytischen Oxydation der Benzolhomologen an Platinanoden und der rein chemischen Oxydation derselben Stoffe fällt am meisten der Umstand auf, dass der anodische Sauerstoff die Seitenkette der aromatischen Kohlenwasserstoffe scheinbar weniger energisch oxydiert, als die Permangansäure oder das Chromtrioxyd. Denn aus Toluol entsteht nach den Angaben der bisherigen Bearbeiter ${ }^{1}$ ) nur Benzaldehyd,und aus dem p-Nitrotoluol gar nur p-Nitrobenzylalkohol ${ }^{2}$ ) an Stelle der erwarteten Carbonsäuren. Aber jedem, mit der äusserst kräftigen Oxydationswirkung des an Platinanoden entwickelten Sauerstoffs vertrauten Elektrochemiker musste der Verdacht aufsteigen, dass den Beobachtern der wahre Verlauf der Oxydation verborgen blieb, und dass die Erklärung nicht, wie vorgeschlagen ${ }^{3}$ ),

1) H. D. Law und F. M. Perkin, Faraday, 1, 31 (1904); Ch. N. 92, 66 (1905); Soc. 91, 258 (1907).

2) K. Elbs, Z. El. Ch. 2, 522 (1896).

3) L. Moser, Elektrolytische Prozesse der org. Chemie, S. 73 (1910). 